\title{
The Use of Sodium Pyrophosphate to Improve a Translucent Clay Simulate
}

\author{
Ryan D. Beemer ${ }^{1}$, S.M. ASCE, Elliese Shaughnessy², S.M. ASCE, Kristen R. Ewert ${ }^{2}$, \\ S.M. ASCE, Nicholas Boardman², S.M. ASCE, Dr. Giovanna Biscontin ${ }^{3}$, M. ASCE, \\ Dr. Charles P. Aubeny ${ }^{4}$, M. ASCE, P.E., and Francisco J. Grajales ${ }^{5,6}$, S.M. ASCE \\ ${ }^{1}$ Laureate Research Associate, Centre for Offshore Foundation Systems, University of \\ Western Australia, Perth, Australia, ryan.beemer@uwa.edu.au \\ ${ }^{2}$ Undergraduate Student, Zachry Department of Civil Engineering, Texas A\&M \\ University, College Station, TX, 77843-3136 \\ ${ }^{3}$ Lecturer, Department of Engineering, University of Cambridge, Cambridge \\ CB3 0EL, United Kingdom, gb479@cam.ac.uk \\ ${ }^{4}$ Professor, Zachry Department of Civil Engineering, Texas A\&M University, College \\ Station, TX, 77843-3136, caubeny@civil.tamu.edu \\ ${ }^{5}$ Graduate Assistant Lecturer, Zachry Department of Civil Engineering., Texas A\&M \\ University, College Station, TX, 77843-3136, fran09@tamu.edu \\ ${ }^{6}$ Lecturer, Faculty of Civil Engineering, Universidad Tecnologica de Panama, Panama \\ City, Republic of Panama, francisco.grajales@utp.ac.pa
}

ABSTRACT: In the ever expanding quest to understand the nature and behavior of soil, translucent and even transparent media have been developed to serve as soil simulates. These artificial soils can be used in experimental models to make visual measurement of phenomena such as geosystem kinematics, soil mass movement, soil particle motion, and pore fluid flow that would be nearly impossible to obtain in natural opaque soils without expensive equipment or boundary effects. One successful type of translucent clay simulate is lithium sodium magnesium silicate (LNM silicate, commonly referred by the trade name Laponite ${ }^{\circledR}$ ); however, it's low density/high void ratio results in higher than typical permeability, low undrained shear strength, and extremely long consolidation times. Until now, translucent soil simulates of only $4.5 \%$ by mass LNM silicate to total mass have been possible. This paper provides a method for creating mixtures of translucent LNM silicate gel/glass as high as $15 \%$ by mass with the additions of an emulsifier, sodium pyrophosphate decahydrate (SPP), which impedes gelation so additional silicate powder can be added. Further, digital image processing techniques are used to present a relationship between LNM silicate, SPP, and translucency and an analysis of the modified simulate's permeability and consolidation properties, with comparisons to natural clays, is also included. 


\section{INTRODUCTION}

To date there has been significant success in the development of coarse grained and silty transparent soils, specifically silica based materials for experimental modeling (Ezzein and Bathurst 2011; Iskander and Lai 1994; Stainer et al. 2012), but a true translucent clay has been difficult to produce. Lithium sodium magnesium silicate (LNM silicate) is a synthetic clay mineralogically similar to hectorite (Neumann 1965). In the literature, LNM silicate is commonly referred by the trade name Laponite ${ }^{\circledR}$, which is a registered trademark of BYK Additives. It can be used to create a highly translucent gel at concentration of $1-2 \%$ by mass and a Wigner or repulsive glass at concentrations above $2 \%$ by mass (Ruzicka and Zaccarelli 2011). When used in experimental modeling these soil simulates allow for visual inspection of phenomena that opaque soils don't typically allow. Translucent soil have been used by geotechnical engineers in physical modeling for years just for this reason (Beemer and Aubeny 2011; Gill and Lehane 2001; Sadek et al. 2003; Wallace et al. 2015).

Though LNM silicate is a good analog for clayey materials it has had a number of draw backs including: low density, higher than typical permeabilities, and a lower than typical coefficient of consolidations. This has limited its use, with its most successful application being a simulate for soft marine clays (Wallace and Rutherford 2015). If the materials density could be increased, while maintaining translucency, then it could be more useful. It is proposed that this can be done with the addition of sodium pyrophosphate (SPP) to the medium. This additive is used in food processing and has been used to decrease the viscosity of bentonite (Santagata et al. 2014). Similar phosphate compounds have also been added to LNM silicate to increase time to gelation (Mongondry et al. 2004; Ruzicka and Zaccarelli 2011). Viscosity is reduced and arrest time is increased due to the negative ions from the SPP adsorbing to the positive rims of the clay plates preventing them from organizing.

If time to gelation or arrest could be sufficiently increased it could be possible to substantially increase the mass of LNM silicate in the medium and improve its geotechnical properties. This paper examines the use of SPP to chemically densify LNM silicate gel/glass and it was discovered that it is possible create a translucent soil simulate with LNM silicate concentrations of $12-15 \%$, over three times higher than previous limits achieved by geotechnical engineers. It should be noted that Neumann $(1965,1970)$ did use tetrasodium pyrophosphate and electrolytes to create LNM silicate sols with solid contents of $10-15 \%$ for use as a thixotropic base in paints, but only the sol structure was studied extensively.

When dealing with transparent soils the common way to assess material optical properties is to measure its refractive index. This however, requires specialized equipment, refractometers. This paper also proposes using a digital camera and a simple image processing algorithm to quantize clarity of translucent soil simulates.

\section{EXPERIMENTAL SETUP}

\section{Emulsifier Densified Silicate Development - General Sample Preparation}

Samples for assessing translucency were prepared with 320 grams of tap water in $470 \mathrm{ml}$ (16 ounce) clear plastic containers resulting in a sample of LNM silicate 
gel/glass about $6.35 \mathrm{~cm}$ (2.5 in) deep. The concentration of LNM silicate, $C_{S}$, was taken as percent of total mass, Equation 1, as is commonly practiced (Wallace and Rutherford 2015):

$$
C_{s}=\frac{m_{s}}{m_{s}+m_{u s}} \cdot 100
$$

where: $C_{S}$ is percent concentration of LNM silicate, $m_{s}$ is mass of LNM silicate powder, and $m_{w}$ is mass of water

The concentration of the SPP additive was taken as mass SPP to mass water:

$$
C_{u p p}=\frac{m_{a p p}}{m_{w}} \cdot 100
$$

where: $C_{S P P}$ is percent concentration of SPP and $m_{S P P}$ is mass of SPP

The ionic strength of water is known to have an effect on the arrest time, and therefore translucency of LNM silicate; in distilled water LNM silicate gel times can be on the order of months (Ruzicka and Zaccarelli 2011). For the purpose of geotechnical sample preparation this is an undesirable trait; therefore, tap water was used instead. The tap water at Texas A\&M University has an ionic strength of $9.7 \times 10^{-}$

${ }^{3} \mathrm{M}$ which falls well below the phase separation area of LNM silicate phase diagrams (Ruzicka and Zaccarelli 2011); meaning a translucent gel or glass should be achieveable with Texas A\&M University tap water.

Samples were prepared by dissolving the SPP thoroughly in water agitated by a high speed mixer. Then LNM silicate powder (BYK Additives 2014) was slowly introduced to the solution and mixed for a total of 5 minutes. The manufacturer recommends mixing LNM silicate powder for a total of 20 minutes (BYK Additives 2014); however, we qualitatively found that 5 minutes of mixing was sufficient with a high speed mixer. After mixing the medium was immediately capped to prevent desiccation and was allowed to sit until it gelled/arrested (did not flow under gravity). The sample was then placed over a background image and photographed with a digital camera from a $28 \mathrm{~cm}$ (11 inch) stand.

The experiments were started at a LNM silicate concentration, $C_{S}$, of $4 \%$. Though mixtures above 3-4\% LNM silicate glass are not recommended (BYK Additives 2014), Wallace and Rutherford (2015) did succeed in creating mixtures of $4.5 \%$ silicate. The quantity of SPP was incrementally increased in each new mixture until there, subjectively, was no blurring of the logo and no bubbles present, Figure 1.

\section{Image Processing}

Material translucency was quantized using a simple image processing technique. Images of the LNM silicate mixtures were compared to a reference image, Figure 2. This was created by placing the previously mentioned $470 \mathrm{ml}$ container filled with $6.36 \mathrm{~cm}$ of water over the sample image. It is assumed that the LNM silicate mixture will not be more translucent than water. The image processing algorithm was 
developed to assess differences between the sample image and reference image caused by bubbling or phase separation and follows:

1. All images convert to grayscale

2. Input image inverted and added to sample image resulting in Image $A, I_{A}$

a. If summed images are identical $\mathrm{I}_{\mathrm{A}}$ will be white ( 1 in grayscale)

3. All entries in Image A summed, $\Sigma \mathrm{I}_{\mathrm{A}}$

4. Similarity Index was calculated as percent difference between $\Sigma I_{A}$ and the sum of a white image of the same size
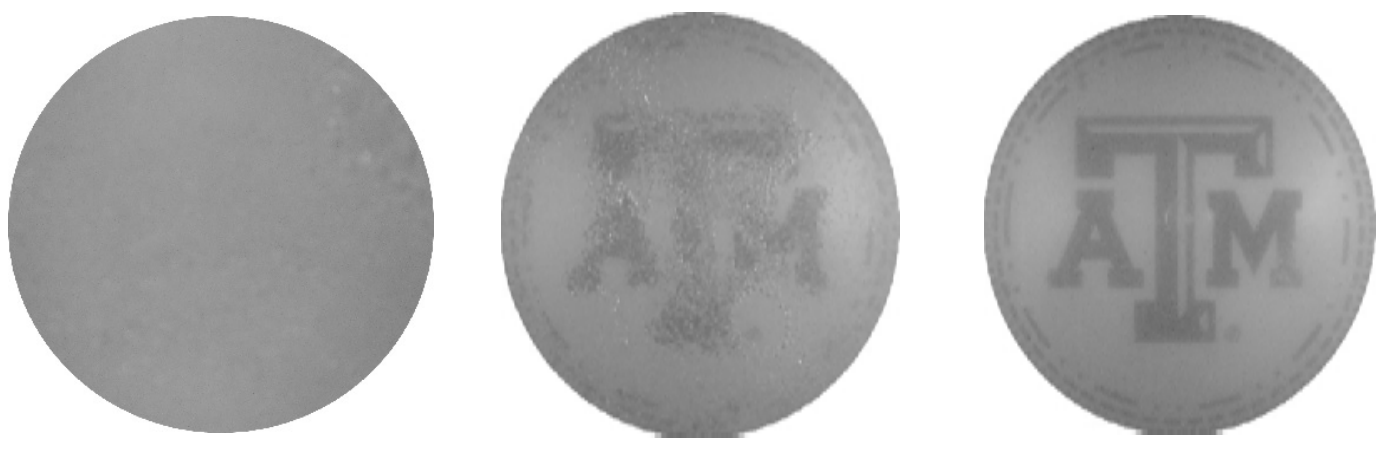

FIG. 1. Increasing translucency of samples with silicate concentration of $12 \%$ and SPP concentrations of $0.70 \%, 0.96 \%$, and $1.5 \%$ (left to right)

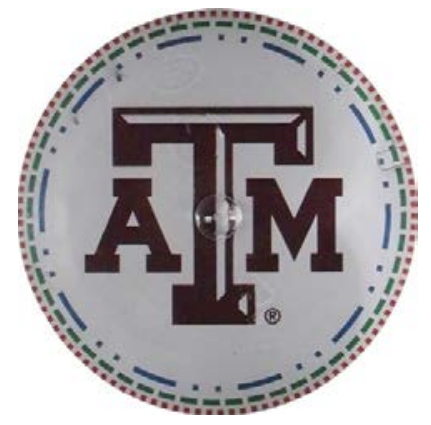

FIG. 2. Sample image for Similarity Index processing

A qualitative assessment is needed to ascertain the minimum Similarity Index of a sufficiently translucent simulate. This can be done by visually inspection, as in Figure 1. Additionally, this algorithms simplicity leads to a number of limitations. This algorithm uses pixel value to assess structural changes in the image; therefore, anything that can vary pixel value will also impact the Similarity Index. This include changes in lighting, use of different cameras (specifically the use of different image sensors), and any post processing of the image.

\section{Consolidation Tests}

In total four consolidation experiments were conducted. LNM silicate concentration, $C_{S}$, and set time (sample rest time after mixing) were varied between 
the experiments as outline in Table 1. Minimum SPP concentrations from the LNM Silicate-SPP curve in Figure 3 were used to create the samples. All were prepared using the above method; however, instead of a clear plastic container they were poured into a 3D printed split mold. The molds were then submerged in tap water to prevent desiccation. Water was allowed to diffusion in (or out) of the sample from holes in the base and lid of the mold, the holes were covered with $20 \mu \mathrm{m}$ filter paper. Set time was taken as time between mixing and start of testing.

The consolidation tests were conducted according to ASTM D2435. The load was doubled each day for five days to reach a maximum loading of $1 \mathrm{~kg}$ before it was unloaded for two days. Unloading results are not presented in this paper.

Table 1. Consolidation Experiments

\begin{tabular}{|c|c|c|}
\hline Test (\#) & LNM silicate Concentration, $\boldsymbol{C}_{\boldsymbol{S}}$, (\%) & Set Time (weeks) \\
\hline 1 & 10 & 3 \\
\hline 2 & 10 & 4 \\
\hline 3 & 15 & 2 \\
\hline 4 & 15 & 4 \\
\hline
\end{tabular}

\section{Permeability Tests}

A total of nine falling head permeability tests were conducted for various concentrations of LNM silicate and set times, Table 2. Minimum SPP concentrations from the LNM Silicate-SPP curve in Figure 3 were used to create the samples. Permeability tests followed ASTM D5084 Method-B. Samples were prepared from the mix procedure above; however, the samples were transferred to a rigid permeability cells prior to arresting, so a flexible membrane was not used. Additionally, $20 \mu \mathrm{m}$ filter paper was placed over the porous stones. The inlet and outlet hoses were filled with tap water to ensure the sample did not desiccate while they set.

Table 2. Falling Head Permeability Experiments

\begin{tabular}{|c|c|c|}
\hline Test (\#) & LNM silicate Concentration, $\boldsymbol{C}_{\boldsymbol{S}}, \mathbf{( \% )}$ & Set Time (weeks) \\
\hline A & 4 & 1 \\
\hline B & 10 & 1 \\
\hline C & 15 & 1 \\
\hline D & 4 & 2 \\
\hline E & 10 & 2 \\
\hline F & 15 & 2 \\
\hline G & 4 & 4 \\
\hline H & 10 & 4 \\
\hline I & 15 & 4 \\
\hline
\end{tabular}




\section{RESULTS AND DISCUSSION}

\section{LNM Silicate-SPP Curve}

The results for minimum SPP concentration to create a translucent LNM silicate mixture, with tap water that has an ionic strength of $9.7 \times 10^{-3} \mathrm{M}$, has been compiled into the LNM Silicate-SPP Curve in Figure 3. The curve is composed of four zones. SPP cannot be dissolved in concentrations, $C_{S P P}$, above $10.9 \%$ at room temperature, red line. The Minimum SPP Curve, fitted with a cubic function, presents minimum concentrations of SPP to LNM Silicate to create a translucent material free of bubbles and with minimum phase separation. Translucent materials are possible between the cubic function and maximum SPP line. The red X indicates that a mixture at an LNM silicate concentration, $C_{S}$, of $17 \%$ and a max SPP concentration will contain bubbles and will not be translucent. The gray triangle indicated a zone of unknown behavior. Materials to the right hand side of the chart will not be translucent due to entrained bubbles or excessive phase separation.

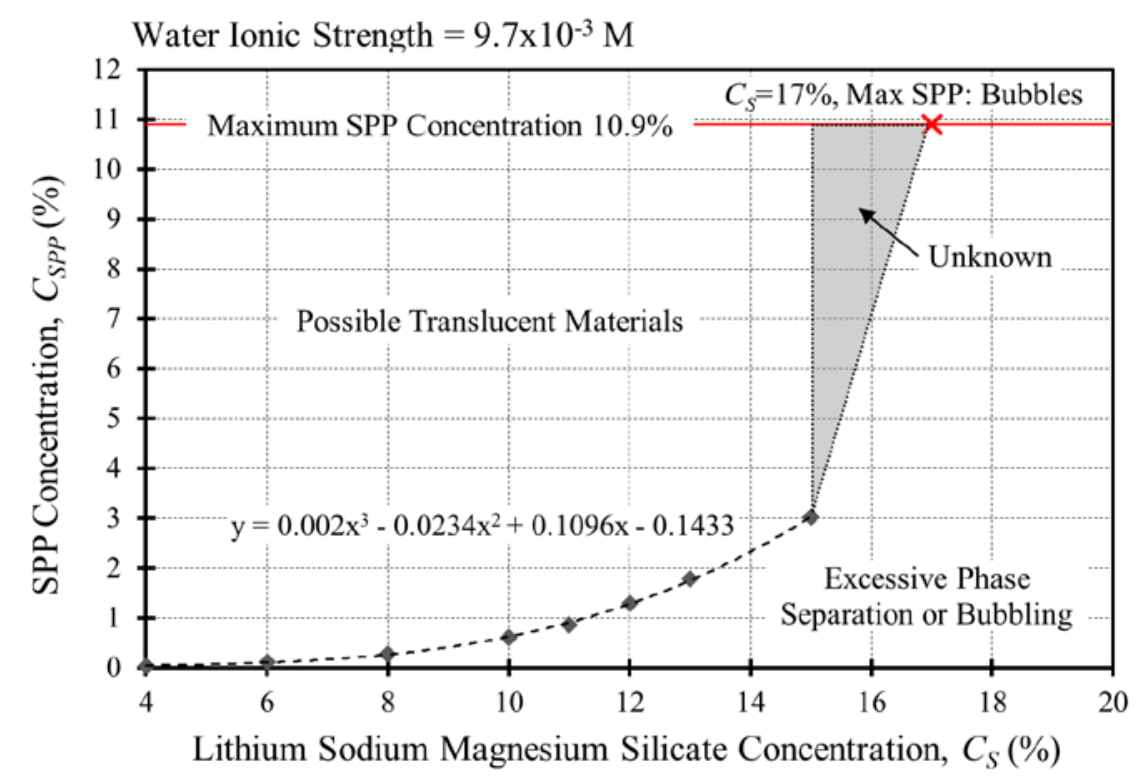

FIG. 3. Design LNM Silicate-SSP curve for translucent clay simulates

The image processing algorithm worked well for quantizing translucency with Similarity Index linearly increasing with clarity, Figure 4. An average Similarity Index of 93.0\% was used to create the Minimum SPP Curve.

\section{Consolidation}

Results from the one dimensional consolidation tests revealed that for a given loading increment, a consolidation period greater than forty-eight hours is necessary for the specimen to reach end of primary consolidation, Figure 5. The 48 hour load increment on that day was accidentally, but did provide valuable insight. Future studies should use ASTM 2435 Method-B which permits a load period of greater than 
twenty four hour. Due to the lack of secondary compression data the final point of the settlement curve was used to calculate the daily coefficient of consolidation, $c_{v}$.

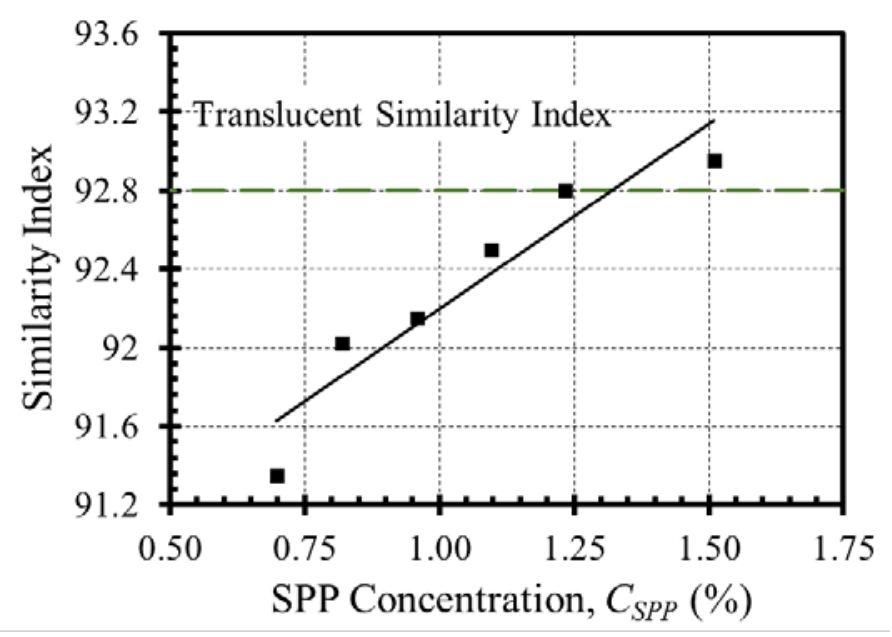

FIG. 4. Plot of qualitative Similarity Index determination for a LNM silicate concentration of $12 \%$, Figure 1

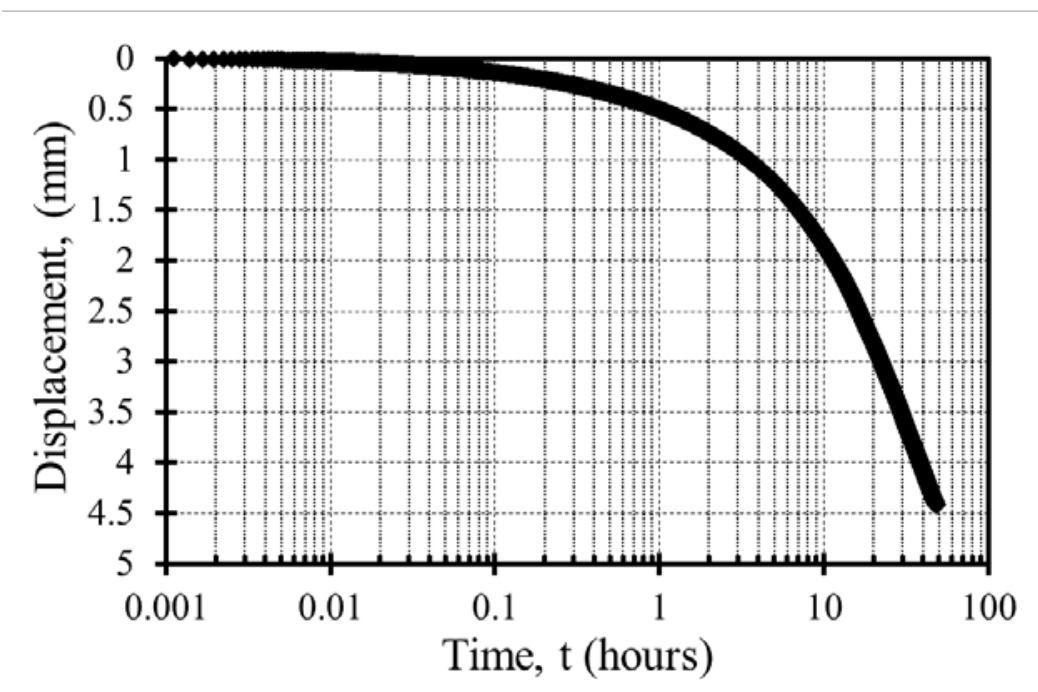

FIG. 5. Example settlement curve, LNM silicate concentration of $10 \%, C_{S}$, 3 week set time

Variation in coefficient of consolidation for both LNM silicate concentration, $C_{S}$, and set time is summarized in Table 3. The coefficient of consolidation increased with set time for LNM silicate concentration, $C_{S}$, of both $10 \%$ and $15 \%$. It should be noted that both specimens that were allowed to set for four weeks experienced failure under the maximum load, $3.10 \mathrm{kPa}$. Results from these experiments are approximately three to five times greater than those measured for lower LNM silicate concentrations by Wallace and Rutherford (2015), $0.017 \mathrm{~m}^{2} / \mathrm{yr}$ for a LNM silicate concentration, $C_{S}$, of $4 \%$. 


\section{Permeability tests}

Hydraulic conductivities from falling head permeability experiments ranged from $7.4 \times 10^{-8} \mathrm{~cm} / \mathrm{s}$ to $5.9 \times 10^{-5} \mathrm{~cm} / \mathrm{s}$, Table 4 and Figure 6a. As a general trend, permeabilities are inversely proportional to concentration of LNM silicate, Cs. When considering set time this initial dataset does not indicate any predictable trends, Figure 6a. However, more testing should be conducted.

Table 3. Coefficient of consolidation for LNM silicate mixtures

\begin{tabular}{|c|c|c|}
\hline $\begin{array}{c}\text { LNM Silicate Concentration, } \\
C_{S},(\%)\end{array}$ & $\begin{array}{l}\text { Set Time } \\
\text { (Weeks) }\end{array}$ & $\begin{array}{c}\text { Coefficient of Consolidation, } \\
c_{v},\left(\mathrm{~m}^{2} / \mathrm{yr}\right)\end{array}$ \\
\hline 10 & 3 & 0.041 \\
\hline 10 & 4 & 0.049 \\
\hline 15 & 2 & 0.049 \\
\hline 15 & 4 & 0.051 \\
\hline
\end{tabular}

Table 4. Falling head permeability results

\begin{tabular}{|c|c|c|c|}
\hline Test (\#) & $\begin{array}{c}\text { Set Time } \\
\text { (weeks) }\end{array}$ & $\begin{array}{c}\text { LNM Silicate } \\
\text { Concentration, } \boldsymbol{C}_{\boldsymbol{s}}, \mathbf{( \% )}\end{array}$ & $\begin{array}{c}\text { Permeability, } \boldsymbol{k}, \\
\text { (cm/s) }\end{array}$ \\
\hline A & 1 & 4 & $4.29 \times 10^{-6}$ \\
\hline B & 1 & 10 & $1.98 \times 10^{-6}$ \\
\hline C & 1 & 15 & $4.57 \times 10^{-7}$ \\
\hline D & 2 & 4 & $5.90 \times 10^{-5}$ \\
\hline E & 2 & 10 & $1.17 \times 10^{-6}$ \\
\hline F & 2 & 15 & $7.37 \times 10^{-8}$ \\
\hline G & 4 & 4 & $2.33 \times 10^{-5}$ \\
\hline H & 4 & 10 & $2.31 \times 10^{-6}$ \\
\hline I & 4 & 15 & $1.23 \times 10^{-7}$ \\
\hline
\end{tabular}

Modified LNM silicate permeability has been compared against data available in the literature, Figure 6b. Mesri and Olson (1971) presented a detailed study on the measurement of permeability of fine grained soils including: kaolinite, illite, and smectite. It can be seen that permeabilities for LNM silicate with SPP compare well with natural soils, with denser LNM silicate concentrations, $C_{S}$, comparing better.

Wallace and Rutherford (2015) reported permeabilities for LNM silicate simulates. They measured permeability using two different laboratory methods: (1) falling head permeability test and (2) incremental consolidation test. Comparison between these and values from this study are presented in Figure 7. Across the board permeabilities of SPP densified clay simulate appear to be one to two orders of magnitude higher than LNM silicate materials formed with just distilled water. 


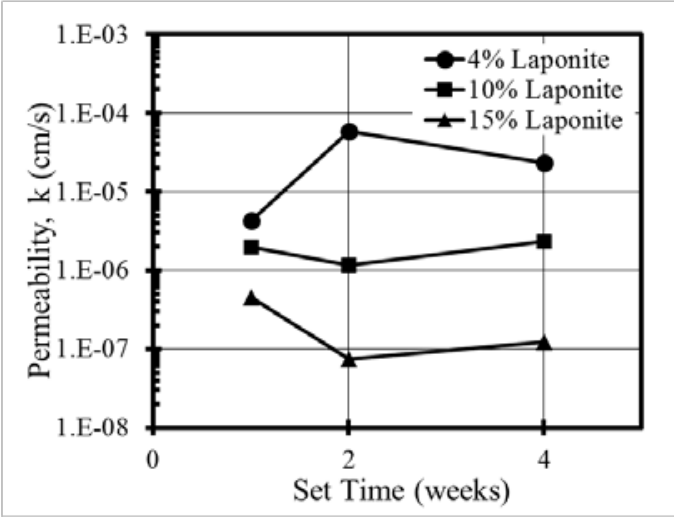

FIG. 6a. Variation of LNM silicate permeabilities

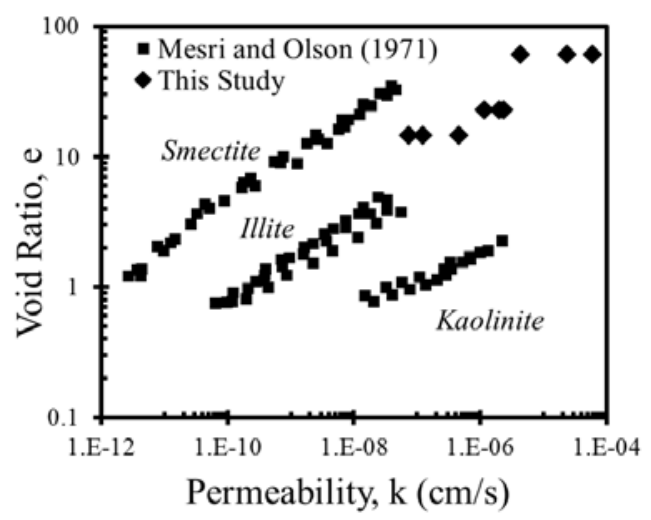

FIG. 6b. Comparison against permeabilities from natural clays

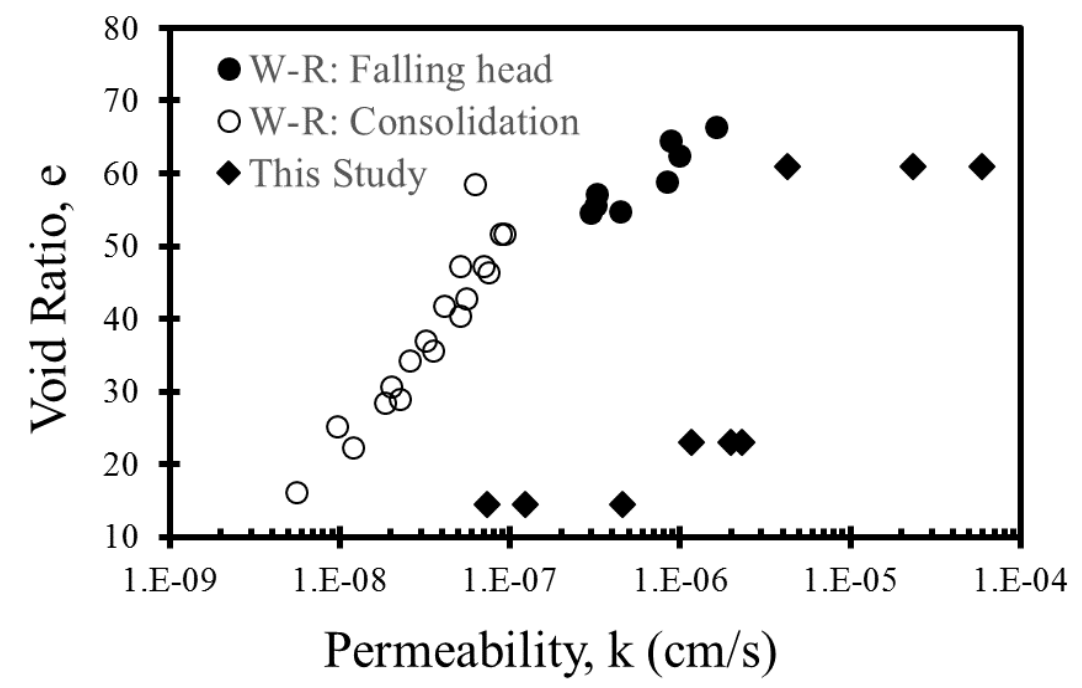

FIG. 7. Comparison to earlier experiments

\section{CONCLUSIONS}

This paper presents a new means of formulating a translucent clay simulate produced with Lithium Sodium Magnesium silicate, Sodium Pyrophosphate, and tap water for geotechnical engineers. These mixtures have LNM silicate concentration 3.33 time higher than previously achieved. Also presented was a simple algorithm for quantizing the translucency of a soil simulates with only a digital camera. Though this algorithm has a number of limitation it is useful for translucency quality control. A LNM Silicate -SPP curve was presented for creating translucent LNM silicate mixtures using tap water with an ionic strength of $9.7 \times 10^{3} \mathrm{M}$. The curve may not be applicable to water with a different ionic strength. Coefficients of consolidation and permeabilities have been measured for the new material. Coefficients of consolidation were found to by three to five time higher than those in previous studies of LNM silicate simulates. While measured permeabilities were found to be one to two orders of magnitude greater than those previously report and compared well to natural clays. 


\section{ACKNOWLEDGEMENTS}

The lead author would like to acknowledge the excellent work of the undergraduate researchers on this project: Elliese Shaughnessy for the laboratory work and MATLAB programming necessary to create the LNM Silicate -SPP curve, Kristen Ewert for conducting and interpreting the consolidation experiments, Nicholas Boardman for mixing the testing specimens and conducting the permeability experiments, and Tom Anderson for assisting with the permeability experiments. We would also like to thank Dr. Cassandra Rutherford for her assistance on the project. This research was funded through the National Science Foundation, Award Number: 1041604.

\section{REFERENCES}

Beemer, R. D., and Aubeny, C. P. (2012). "Digital image processing of drag embedment anchors in translucent silicate gel." GeoManitoba, Canadian Geotechnical Society, Winnepeg.

BYK Additives. (2014). Laponite: performance additives. Wesel, Germany.

Ezzein, F., and Bathurst, R. (2011). "A transparent sand for geotechnical laboratory modeling.” Geotech. Test. J., ASTM, 34 (6): 1-12.

Gill, D., and Lehane, B. (2001). "An optical technique for investigating soil displacement patterns.” Geotech. Test. J., ASTM, 24 (3): 324-329.

Iskander, M., and Lai, J. (1994). "Development of a transparent material to model the geotechnical properties of soils.” Geotech. Test. J., ASTM, 17 (4): 425-433.

Mesri, G. and Olson, R.E. (1971). "Mechanisms controlling the permeability of clays.” Clays and Clay Miner., Clay Mineral Society, 19: 151-158.

Mongondry, P., Nicolai, T., and Tassin, J. F. (2004). "Influence of pyrophosphate or polyethylene oxide on the aggregation and gelation of aqueous laponite dispersions.” J. of Colloid and Interf. Sci., Elsevier, 275 (1): 191-196.

Neumann, B. S. (1965). "Behaviour of a synthetic clay in pigment dispersions." Rheologica Acta, Springer, 4: 250-255.

Neumann, B. S. (1970). "The formation of stable sols from Laponite, a synthetic hectorite-like clay.” Clay Minerals, Clay Mineral Society, 8 (4): 389-404.

Ruzicka, B., and Zaccarelli, E. (2011). "A fresh look at the Laponite phase diagram.” Soft Matter, Royal Society of Chemistry, 7 (4): 1268-1286.

Sadek, S., Iskander, M. G., and Liu, J. (2003). “Accuracy of digital image correlation for measuring deformations in transparent media." J. of Comput. Civil Eng., ASCE, 17 (2): 88-96.

Santagata, M., Clarke, J. P., Bobet, A., Drnevich, V. P., El Mohtar, C. S., Huang, P.T., and Johnston, C. T. (2014). "Rheology of concentrated bentonite dispersions treated with sodium pyrophosphate for application in mitigating earthquakeinduced liquefaction.” Appl. Clay Sci., Elsevier, 99: 24-34.

Stanier, S. A., Black, J. A., and Hird, C. C. (2012). "Enhancing accuracy and precision of transparent synthetic soil modelling.” Int. J. of Phys. Model. Geotech, ICE, 12 (4): 162-175. 
Wallace, J. F., Chini, C., Rutherford, C., and Peschel, J. (2015). "Visualizing the shallow failure mechanism of the T-bar penetrometer.” IFCEE 2016 (GSP 256), ASCE, San Antonio: 330-338.

Wallace, J. F., and Rutherford, C. J. (2015). "Geotechnical properties of Laponite RD®.” Geotech. Test. J., ASTM, 38 (5): 574-587. 